\title{
Recent Progress on 3-D Integrated Intra-Chip Free-Space Optical Interconnect
}

\author{
Berkehan Ciftcioglu, Jing Gao, Rebecca Berman, Manish Jain, Duncan Moore, Gary Wicks, Michael Huang, Eby \\ G. Friedman, and Hui Wu
}

Recently, we proposed an intra-chip interconnect system for future multi-core processors based on free-space optics and 3-D integrated photonic devices [1], [2], [4]. The main objective is to construct an all-to-all communication fabric with high bandwidth density, low latency, and good energy efficiency without routing or switching. As shown in Fig. 1, this free-space optical interconnect (FSOI) system consists of a photonics layer and a free-space optical guiding medium constructed using micro-mirrors and micro-lenses, which are stacked on top of the CMOS electronics layer by 3-D chip integration. Note that this interconnect system can also be used for inter-chip communications.

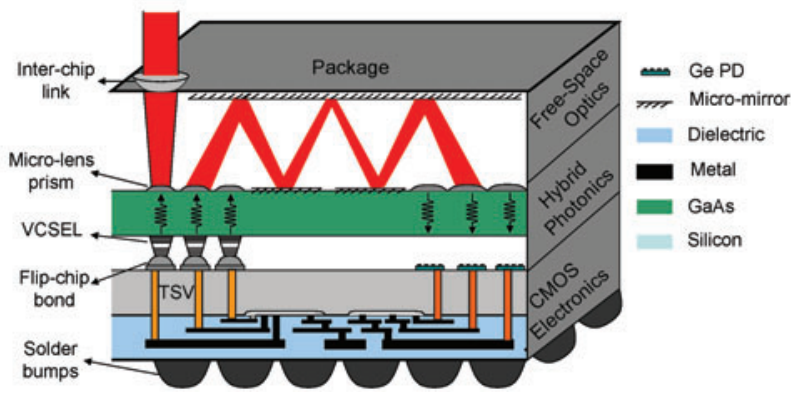

Fig. 1. Cross-sectional view of the proposed intra-chip FSOI system.

Here, we compare the energy efficiency of a WDM-based optical interconnect similar to [3] and the proposed intra-chip FSOI system to illustrate the advantages of our approach. The operation wavelength of the WDM-based systems is chosen to be $1550 \mathrm{~nm}$, while the FSOI system still uses $980 \mathrm{~nm}$. To emphasize the effects of photonic devices and optics design, the transceiver circuits is excluded from the calculation, which would be similar in both cases. To simplify the calculation, the PDs in both systems are assumed to have $100 \%$ quantum efficiency and adequate bandwidth to support 10-Gbps data rate, which gives the WDM system unfair advantage. Our FSOI link loss and energy efficiency is calculated similar to [1], based on the design parameters shown in Table. I. Similarly based

This work was partially supported by NSF grants CCF0829915 and DMR1124601 and the DOE Office of Inertial Confinement Fusion under Cooperative Agreement No. DE-FC52-08NA28302, the University of Rochester, and the New York State Energy Research and Development Authority. The support of DOE does not constitute an endorsement by DOE of the views expressed in this article.

B. Ciftcioglu, M. Huang, E.G. Friedman, and H. Wu are with the Department of Electrical and Computer Engineering, University of Rochester, Rochester, NY 14627 USA(e-mail: hui.wu@ rochester.edu).

R. Berman, Z. Darling, M. Jain, D. Moore, and G. Wicks are with the Institute of Optics, University of Rochester, Rochester, NY 14627 USA. on Table. I, in the WDM-based system, a 0.77-nm wavelength channel spacing and a total of 13 channels in each node are selected to utilize the entire 10-nm free-spectral-range (FSR). Each of these 13 channels experiences an additional 0.066$\mathrm{dB}$ loss due to the contribution from the other 12 adjacent channels in each node. The overall link optical loss for the longest optical path meandering through each row of nodes is calculated by adding $0.3-\mathrm{dB} / \mathrm{cm}$ waveguide loss and 0.05 $\mathrm{dB}$ bend loss to $0.11-\mathrm{dB}$ modulator insertion loss per node, as shown in Fig. 2-a. The corresponding energy efficiencies are plotted in Fig. 2-b with respect to increasing number of nodes. The average electrical power consumed for a single bit WDM system is $6.1 \mathrm{~mW}$, corresponding to $0.61-\mathrm{pJ} / \mathrm{bit}$ at $10-\mathrm{Gbps}$ data rate. The switching energy efficiency of the modulator is $0.183 \mathrm{pJ} / \mathrm{bit}$ and constitutes one third of the total energy consumption. Similarly, a VCSEL in the FSOI system consumes $0.69-\mathrm{mW}$ average power, corresponding to a 0.069 $\mathrm{pJ} /$ bit energy efficiency.

It is noteworthy to mention that the calculations are highly optimistic for the WDM system. First, it does not include the thermal tuning power consumption, required to accurately control the wavelengths of micro-rings. Second, the stateof-the-art WDM laser sources have energy efficiency of a few percentage, much worse than our assumption. Finally and more importantly, silicon micro-ring modulators exhibits insertion loss more than $0.5-\mathrm{dB}$, resulting in over $60-\mathrm{dB}$ optical loss in a single 36 -node link.

TABLE I

Optical and Photonic Device Parameters For a 36 node INTERCONNECT SYSTEM

\begin{tabular}{|c|c|c|c|}
\hline \multicolumn{2}{|c|}{ VCSEL } & \multicolumn{2}{|c|}{ Micro-ring Modulator } \\
\hline $\bar{\lambda}_{0}$ & $980 \mathrm{~nm}$ & $\lambda_{0}$ & $1550 \mathrm{~nm}$ \\
\hline $\mathrm{f}_{3 d B}$ & $13 \mathrm{GHz}$ & $\mathrm{f}_{3 d B}$ & $9.6 \mathrm{GHz}$ \\
\hline$\eta_{\text {Slope }}$ & $0.67 \mathrm{~W} / \mathrm{A}$ & FSR & $10 \mathrm{~nm}$ \\
\hline$\eta_{\text {Wallplug }}$ & $30 \%$ & Q & 20000 \\
\hline $\mathrm{I}_{t h}$ & $0.14 \mathrm{~mA}$ & $\delta \lambda$ & $0.07 \mathrm{~nm}$ \\
\hline $\mathrm{I}_{o n}$ & $0.47 \mathrm{~mA}$ & $\Delta \lambda$ & $0.383 \mathrm{~nm}$ \\
\hline $\mathrm{V}_{f}$ & $1.62 \mathrm{~V}$ & Loss & $0.0437 \mathrm{~dB}$ \\
\hline Energy & $69 \mathrm{fJ} / \mathrm{bit}$ & $\mathrm{V}_{r}$ & $7.5 \mathrm{~V}$ \\
\hline & & $\mathrm{C}_{j}$ & $6.6 \mathrm{fF}$ \\
\hline & & Energy & $183 \mathrm{fJ} / \mathrm{bit}$ \\
\hline \multicolumn{2}{|c|}{ PD in FSOI } & \multicolumn{2}{|c|}{ PD in WDM } \\
\hline$\eta_{\text {quantum }}$ & $100 \%$ & $\eta_{\text {quantum }}$ & $100 \%$ \\
\hline $\mathrm{R}_{P D}$ & $0.79 \mathrm{~A} / \mathrm{W}$ & $\mathrm{R}_{P D}$ & $1.247 \mathrm{~A} / \mathrm{W}$ \\
\hline \multicolumn{2}{|c|}{ Micro-lens } & \multicolumn{2}{|c|}{ Waveguide } \\
\hline Loss & $0.1 \mathrm{~dB}$ & Loss & $0.3 \mathrm{~dB} / \mathrm{cm}$ \\
\hline
\end{tabular}

A new FSOI prototype was implemented with a complete set of fabricated devices. The germanium metal-semiconductormetal (MSM) PD is an improved version of [5], and exhibits over $12-\mathrm{GHz}$ bandwidth, $0.315-\mathrm{A} / \mathrm{W}$ responsivity and $7-\mu \mathrm{A}$ 


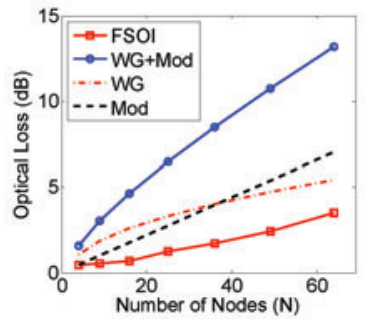

(a)

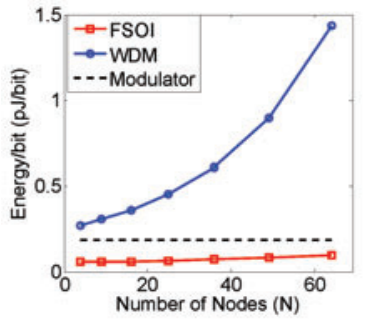

(b)
Fig. 2. The calculated (a) optical loss and (b) energy efficiency of the FSOI and WDM-based optical interconnect systems with respect to increasing number of nodes. The breakdown of the loss components for the WDM system is shown with dash lines. In this calculation, the transceiver electronics (laser/modulator driver and receiver) are not included in the total power consumption.

dark current. The micro-lenses are fabricated on a $525-\mu \mathrm{m}$ thick fused silica substrate via melt-and-reflow technique. The VCSEL is fabricated on a $625-\mu \mathrm{m}$ thick GaAs/AlGaAs epitaxial wafer grown by IQE, designed for 850-nm operation. The VCSEL, shown in Fig. 3-a, has a 0.5-mA threshold current, $0.67-\mathrm{A} / \mathrm{W}$ slope efficiency, and $26.8 \%$ wall-plug efficiency at 1-mW optical power (Fig. 3-b). The measured full-width halfmaximum divergence angle is $19.2^{\circ}$. The pitch size between micro-lenses, PDs and VCSELs are chosen as $250 \mu \mathrm{m}$. To facilitate wirebonding VCSELs and PDs to the printed circuit board, silica spacers are inserted between the micro-lenses and the VCSEL/PDs.

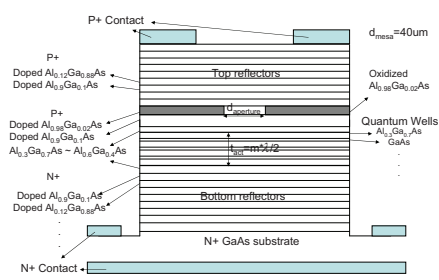

(a)

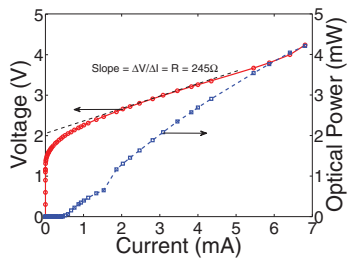

(b)
Fig. 3. (a) The fabricated VCSEL structure on a commercially grown GaAs substrate. (b) The dc measurement results of the fabricated $8-\mu \mathrm{m}$ aperture size VCSEL.

Similar to [1], the prototype is implemented on a PCB, as shown in Fig. 4. After the VCSELs are wirebonded to the $\mathrm{PCB}$, a $200-\mu \mathrm{m}$ thick fused silica glass spacer is placed and glued on top of the chip using non-conductive epoxy. Then the micro-lenses with an $200 \mu \mathrm{m}$ aperture size and a focal point $210 \mu \mathrm{m}$ away from the backside of the lens is glued on top. The PD chip is similarly integrated with another microlens chip. For testing purpose, $95 \%$ reflective mirrors with a 45-degree angle are mounted on top of each assembled chip stack approximately $1 \mathrm{~mm}$ from the surface of the lenses.

To evaluate the dc properties, both the optical transmission for a specific link and the optical crosstalk of adjacent links are measured with respect to distance. As shown in Fig. 5-a, at a $0.65-\mathrm{mW}$ laser optical power and 1.5-mA VCSEL bias current, the transmission loss is $8.9 \mathrm{~dB}$ and $9.8 \mathrm{~dB}$ at $30-\mathrm{mm}$ and $35-\mathrm{mm}$ distances, respectively. The crosstalk power is $30 \mathrm{~dB}$ at $30 \mathrm{~mm}$ and increases to $-28 \mathrm{~dB}$ at $35 \mathrm{~mm}$. Figure 5-b shows the small-signal bandwidth of the link. The bandwidth
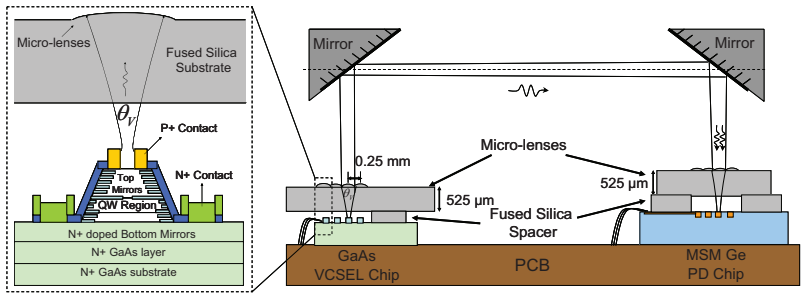

(a)

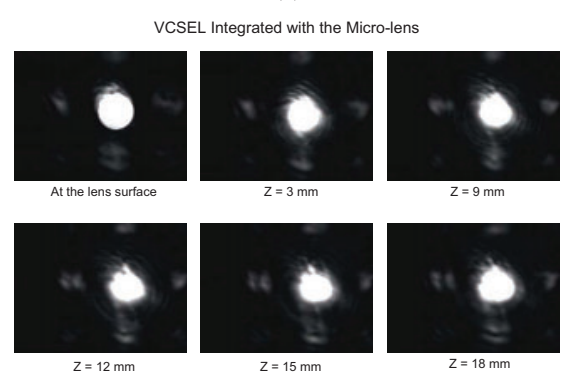

(b)

Fig. 4. (a) 2-D cross-section schematic, and (b) image of the beam collimated by VCSEL lens at different distances. The beam size shrinks down up to 1.5$\mathrm{cm}$ distance and grows beyond. The measured beam waist after the lens is at $1.6-\mathrm{cm}$.

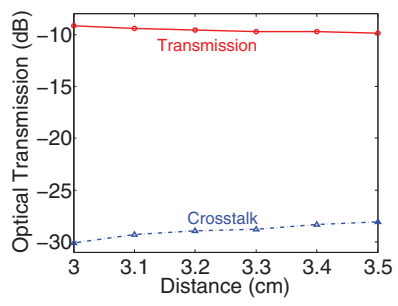

(a)

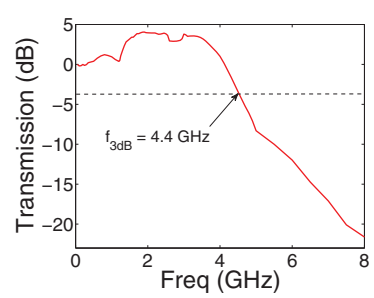

(b)
Fig. 5. (a) Transmitted signal and crosstalk powers at different distances. (b) Small-signal bandwidth measured at $3 \mathrm{~cm}$.

is measured as $4.4 \mathrm{GHz}$, limited by the wirebonds and the VCSEL bandwidth. It can be further improved by increasing the aperture size and/or shrinking the device size, and hence reducing the series resistance and parasitic capacitance.

\section{REFERENCES}

[1] B. Ciftcioglu et al., "A 3-D integrated intra-chip free-space optical interconnect for many-core chips," IEEE Photonics Technology Letters, 23(3):164-166, (Feb. 2011).

[2] B. Ciftcioglu et al., "3-D integrated heterogeneous intra-chip free-space optical interconnect," Optics Express, 20(4):4331-4345, (Feb. 2012).

[3] R. G. Beausoleil et al., "A nanophotonic interconnect for highperformance many-core computation," In 16th IEEE Symposium on High Performance Interconnects, HOTI '08, pages 182-189, (Aug. 2008).

[4] J. Xue et al., "An intra-chip free-space optical interconnect," 37th International Symposium on Computer Architecture ISCA, pages 94105, (2010).

[5] B. Ciftcioglu et al., "An 850-nm normal-incidence germanium metalsemiconductor-metal photodetector with $13-\mathrm{GHz}$ bandwidth and $8-\mu \mathrm{A}$ dark current," IEEE Photonics Technology Letters, 22(24):1851-1853, (Dec. 2010). 